\title{
Estimation of orbit change and emittance growth due to random misalignment in long linacs
}

\author{
Kiyoshi Kubo \\ KEK, High Energy Accelerator Research Organization, 1-1 Oho, Tsukuba, Ibaraki 305-0801, Japan
}

(Received 15 September 2010; published 4 January 2011)

\begin{abstract}
In linear accelerators, the transverse beam orbit is induced by tilts of accelerating cavities (deviation of accelerating field direction from designed beam direction) and transverse offset of quadrupole magnets. Estimating induced orbit and emittance growth due to such random errors is important for evaluation of performance of linacs, especially where stable and low emittance beam is required, such as linear colliders. Usually, such estimations are performed using tracking simulations, including the Monte Carlo method, which tends to take a long time. Here, a much faster and simpler method of quantitative estimation of beam orbit and emittance growth is reported. This method is valid for very long linacs with many components, where statistical treatment of errors is justified. It is shown that the results from the method agree well with tracking simulations for the ILC (International Linear Collider) main linac.
\end{abstract}

DOI: 10.1103/PhysRevSTAB.14.014401

PACS numbers: 29.27.Bd

\section{INTRODUCTION}

In linear accelerators, the transverse beam orbit is induced by tilts of accelerating cavities (deviation of accelerating field direction from designed beam direction) and transverse offset misalignment of quadrupole magnets. As for linear colliders, in cases where very stable and low emittance beam is required, estimating induced orbit and emittance growth due to such random errors is important for evaluation of performance of the linacs.

Usually, such estimation is performed using tracking simulations including the Monte Carlo method, with many different sets of random numbers, which tend to take a long time.

In the following sections we derive formulas of beam orbit and emittance growth due to cavity tilt and quadrupole magnet offset. Then, results are compared with tracking simulations for the ILC main linac. For simplicity, we only consider one transverse direction, denoting $y$. It is straightforward to include the other direction. Also, we do not consider effects of wakefield in this report.

There were various past studies on analytic and semianalytic estimations of orbit change due to random misalignments in high energy linacs (for example, [1-6]). Our method for beam orbit is basically the same as in these past works and gives similar formulas. However, our method and formulas for emittance growth are new. Reference [1] extensively studied analytic estimation of emittance growth in high energy linear accelerators. Though it gave a formula of emittance growth induced by cavity tilt, it did not consider beam energy spread, which is dominantly important in the ILC main linac. This reference also gave emittance growth with correlated misalignment of magnets and emittance after orbit corrections, but no formula for random misalignment of quadrupole magnets was given. Reference [3] gives expressions of chromatic dependence of the orbit with random misalignment of quadrupole magnets. However, its assumption is not valid in the case of the ILC main linac.

\section{EFFECT OF TILT OF ACCELERATING CAVITY}

\section{A. Transverse kick by one cavity with tilt angle}

In a linac, if a cavity axis deviates from the designed beam direction, beam particles are kicked transversely by the accelerating field of the cavity.

For ultrarelativistic beam, the transverse momentum change in a cavity with voltage $V_{c}$ and tilt angle $\theta$ can be expressed as

$$
\Delta p_{y, \text { in }}=\frac{e V_{c}}{c} \sin \theta
$$

We should also consider edge fields of the cavity. (The effect of the edge field can be derived applying Maxwell's equations to field of an accelerating structure. See, for example, [7].) Using a hard edge model, transverse momentum change at the entrance and the exit of a cavity can be expressed as

$$
\Delta p_{y, \text { ent }}=-y_{\text {ent }} \frac{e V_{c}}{2 L c}
$$

and

$$
\Delta p_{y, \mathrm{ext}}=y_{\mathrm{ext}} \frac{e V_{c}}{2 L c},
$$

respectively, where $y_{\text {ent }}$ and $y_{\text {ext }}$ are transverse offset of the particle with respect to the cavity center, and $L$ is length of the cavity. Then,

$$
\Delta p_{y, \text { ent }}+\Delta p_{y, \text { ext }}=-\left(y_{\text {ent }}-y_{\text {ext }}\right) \frac{e V_{c}}{2 L c} .
$$

Assuming the beam orbit angle is negligibly small compared with the cavity tilt angle,

$$
y_{\mathrm{ent}}-y_{\mathrm{ext}}=L \sin \theta,
$$


and

$$
\Delta p_{y, \text { ent }}+\Delta p_{y, \text { ext }}=-\frac{e V_{c}}{2 c} \sin \theta=-\frac{1}{2} \Delta p_{y, \text { in }}
$$

It means the edge fields reduce the transverse momentum change by a half of that from the field inside the cavity. As total momentum change, we have

$$
\Delta p_{y}=\frac{e V_{c}}{2 c} \sin \theta
$$

Then, transverse angle change (kick angle) is transverse momentum change divided by $E / c$ for ultrarelativistic particles,

$$
\Theta=\frac{e V_{c}}{2 E} \sin \theta \approx \frac{e V_{c} \theta}{2 E},
$$

where $E$ is the beam energy at the cavity.

\section{B. Orbit due to random tilt of many cavities}

Summing up effects of all cavities in a linac, the vertical position and angle at the end of linac can be expressed as

$$
\begin{aligned}
& y_{f}=\sum_{i} \sqrt{\beta_{i} \beta_{f}} \sin \phi_{i} \sqrt{E_{i} / E_{f}} \Theta_{i}, \\
& y_{f}^{\prime}=\sum_{i} \sqrt{\beta_{i} / \beta_{f}}\left(\cos \phi_{i}-\alpha_{f} \sin \phi_{i}\right) \sqrt{E_{i} / E_{f}} \Theta_{i},
\end{aligned}
$$

where the summation is taken for all cavities in the linac, $\alpha_{f}$ and $\beta_{f}$ are the alpha and beta function at the end of linac, $\beta_{i}$ is the beta function at the $i$ th cavity, $\phi_{i}$ is the betatron phase advance between the $i$ th cavity to the linac end, $E_{i}$ is the beam energy at the $i$ th cavity, $E_{f}$ is the beam energy at the end of linac, and $\Theta_{i}$ is the kick angle at the $i$ th cavity. The factor $\sqrt{E_{i} / E_{f}}$ represents adiabatic damping due to acceleration.

Assuming $\Theta_{i}$ is random and independent for each cavity, expected (average) action at the linac end is

$$
\begin{aligned}
\left\langle J_{f}\right\rangle & \equiv\left\langle\left(\gamma_{f} y_{f}^{2}+2 \alpha_{f} y_{f} y_{f}^{\prime}+\beta_{f} y_{f}^{2}\right) / 2\right\rangle \\
& =\sum_{i} \beta_{i} E_{i} / E_{f}\left\langle\Theta_{i}^{2}\right\rangle / 2 \\
& =\sum_{i} \beta_{i} E_{i} / E_{f}\left\langle\left(e V_{c} \theta_{i} / 2 E_{i}\right)^{2}\right\rangle / 2 \\
& =\frac{1}{8 E_{f}} \sum_{i} \frac{\left(e V_{c}\right)^{2} \beta_{i}\left\langle\theta_{i}^{2}\right\rangle}{E_{i}},
\end{aligned}
$$

where \langle\rangle denote average over many sets of random errors and $\gamma_{f} \equiv\left(1+\alpha_{f}^{2}\right) / \beta_{f}$. We used

$$
\left\langle\theta_{i} \theta_{j}\right\rangle=\delta_{i j}\left\langle\theta_{i}^{2}\right\rangle .
$$

This formula is suitable for numerical calculation to estimate expected orbit for a given design of a linac, using a computer.
TABLE I. List of assumptions and approximations for analytic formulas.

Same mean square of tilt angle for all cavities

Same mean square of offset for all quadrupole magnets

Energy deviation, $\delta E$, is constant for each particle

Large number of components, for taking averages.

Uniform FODO lattice

Same energy gain in all cavities

Take lowest order of energy spread, $\sigma_{E}^{2}$, for emittance growth

Here, we derive a more simple expression with some approximations. For a long linac with many cavities with the same accelerating voltage, assuming beta function does not depend on the beam energy, we can take averages of $\beta_{i}$, $E_{i}$, and $\left\langle\theta_{i}^{2}\right\rangle$ separately. We also assume the expected tilt angle square is the same for all cavities. Then, we can have

$$
\beta_{i} \rightarrow \bar{\beta}, \quad\left\langle\theta_{i}^{2}\right\rangle \rightarrow\left\langle\theta^{2}\right\rangle,
$$

where $\bar{\beta}$ is the average of the beta function. And

$$
\sum_{i} \frac{1}{E_{i}} \rightarrow \int_{E_{0}}^{E_{f}} \frac{d E}{e V_{c} E}=\frac{1}{e V_{c}} \log \left(E_{f} / E_{0}\right)
$$

where $E_{0}$ is the initial beam energy. Then,

$$
\left\langle J_{f}\right\rangle \approx \frac{e V_{c}}{8 E_{f}} \bar{\beta} \log \left(E_{f} / E_{0}\right)\left\langle\theta^{2}\right\rangle .
$$

It is convenient to multiply this by $\gamma$ (energy factor) for comparison with normalized emittance,

$$
\gamma\left\langle J_{f}\right\rangle \approx \frac{e V_{c}}{8 m c^{2}} \bar{\beta} \log \left(E_{f} / E_{0}\right)\left\langle\theta^{2}\right\rangle .
$$

Here, we have obtained a simple formula for expected orbit change due to random tilt angle of accelerating cavities. Assumptions and approximations used in this article are listed in Table I. Some of them will be used later.

\section{Emittance growth due to random tilt of many cavities}

Here, we consider emittance growth from the dispersive effect. Effects of wakefield are ignored, which are usually not very important for superconducting cavities using relatively low rf frequencies, such as for ILC.

Dispersive effect is estimated from orbit difference between particles with different energies. Let us assume that position and angle deviation are proportional to energy deviation, as follow:

$$
\delta y=\eta \delta E / E \quad \delta y^{\prime}=\eta^{\prime} \delta E / E,
$$

where $\delta E / E$ is relative energy deviation of a particle, and $\eta$ and $\eta^{\prime}$ are dispersion and angle dispersion, respectively. The square of emittance with such deviation is expressed as 


$$
\begin{aligned}
\epsilon^{2}= & \overline{(y-\bar{y})^{2}} \times \overline{\left(y^{\prime}-\overline{y^{\prime}}\right)^{2}}-\left[\overline{(y-\bar{y})\left(y^{\prime}-\overline{y^{\prime}}\right)}\right]^{2} \\
= & \overline{\left(y_{0}+\delta y-\overline{y_{0}}-\overline{\delta y}\right)^{2}} \times \overline{\left(y_{0}^{\prime}+\delta y^{\prime}-\overline{y_{0}^{\prime}}-\overline{\delta y^{\prime}}\right)^{2}} \\
& -\left[\overline{\left(y_{0}+\delta y-\overline{y_{0}}-\overline{\delta y}\right)\left(y_{0}^{\prime}+\delta y^{\prime}-\overline{y_{0}^{\prime}}-\overline{\delta y^{\prime}}\right)}\right]^{2} \\
= & \epsilon_{0}^{2}+\epsilon_{0}\left[\gamma_{y}(\delta y)^{2}+2 \alpha_{y} \delta y \delta y^{\prime}+\beta_{y}\left(\delta y^{\prime}\right)^{2}\right] \\
= & \epsilon_{0}^{2}+\epsilon_{0}\left[\gamma_{y}(\eta)^{2}+2 \alpha_{y} \eta \eta^{\prime}+\beta_{y}\left(\eta^{\prime}\right)^{2}\right] \overline{(\delta E / E)^{2}},
\end{aligned}
$$

where overlines denote average over all particles, $y_{0}, y_{0}^{\prime}$, and $\epsilon_{0}$ are position, angle, and emittance without deviation due to the dispersion, $\alpha_{y}, \beta_{y}$, and $\gamma_{y}$ are Twiss parameters.

So, for evaluating emittance growth, we calculate $\left[\gamma_{y}(\delta y)^{2}+2 \alpha_{y} \delta y \delta y^{\prime}+\beta_{y}\left(\delta y^{\prime}\right)^{2}\right]$ or $\left[\gamma_{y}(\eta)^{2}+2 \alpha_{y} \eta \eta^{\prime}+\right.$ $\left.\beta_{y}\left(\eta^{\prime}\right)^{2}\right]$, at the end of the linac.

First, angle change due to cavity tilt is proportional to the inverse of particle energy. Therefore, for orbit difference due to energy difference, we replace $\Theta$ in Eq. (8) by $(-\delta E / E) \Theta$, assuming $\delta E<E$. Then,

$$
\begin{aligned}
\delta y_{f, 1}= & \sum_{i} \sqrt{\beta_{i} \beta_{f}} \sin \phi_{i} \sqrt{E_{i} / E_{f}}\left(-\delta E / E_{i}\right) \Theta_{i}, \\
\delta y_{f, 1}^{\prime}= & \sum_{i} \sqrt{\beta_{i} / \beta_{f}}\left(\cos \phi_{i}-\alpha_{f} \sin \phi_{i}\right) \\
& \times \sqrt{E_{i} / E_{f}}\left(-\delta E / E_{i}\right) \Theta_{i} .
\end{aligned}
$$

There is another effect to be considered. A particle going through a quadrupole magnet with offset is transversely kicked by angle

$$
\theta_{q}=k_{q} y_{q} \frac{E_{q}}{E_{q}+\delta E_{q}}
$$

where $k_{q}$ is integrated strength of the quadrupole magnet, $y_{q}$ particle position at the magnet, $E_{q}$ the nominal beam energy at the magnet, and $\delta E_{q}$ the energy deviation of the particle at the magnet. For $\left|\delta E_{q}\right| \ll E_{q}$, kick angle difference due to the energy deviation is

$$
\delta \theta_{q}=k_{q} y_{q} \frac{-\delta E_{q}}{E_{q}} .
$$

Then, orbit and angle difference at the end of the linac due to this effect is

$$
\begin{aligned}
\delta y_{f, 2}= & \sum_{q} \sqrt{\beta_{f} \beta_{q}} \sin \phi_{q} \sqrt{E_{q} / E_{f}} k_{q} y_{q} \frac{-\delta E_{q}}{E_{q}}, \\
\delta y_{f, 2}^{\prime}= & \sum_{q} \sqrt{\beta_{q} / \beta_{f}}\left(\cos \phi_{q}-\alpha_{f} \sin \phi_{q}\right) \\
& \times \sqrt{E_{q} / E_{f}} k_{q} y_{q} \frac{-\delta E_{q}}{E_{q}},
\end{aligned}
$$

where the summation is taken for all quadrupole magnets in the linac, $\beta_{q}$ is the beta function at each quadrupole magnet, and $\phi_{q}$ is the phase advance between each quadrupole magnet to the linac end. From Eq. (9),

$$
y_{q}=\sum_{i<q} \sqrt{\beta_{q} \beta_{i}} \sin \phi_{i q} \sqrt{E_{i} / E_{q}} \Theta_{i}
$$

where the summation is taken for all cavities upstream of the quadrupole magnet, and $\phi_{i q}$ is the phase advance between the $i$ th cavity to the quadrupole magnet.

For the total orbit difference of the first order of energy deviation, Eq. (18) and (21) should be added:

$$
\delta y_{f}=\delta y_{f, 1}+\delta y_{f, 2}, \quad \delta y_{f}^{\prime}=\delta y_{f, 1}^{\prime}+\delta y_{f, 2}^{\prime} .
$$

We take the average of $\gamma_{f}\left(\delta y_{f}\right)^{2}+2 \alpha_{f} \delta y_{f} \delta y_{f}^{\prime}+$ $\beta_{f}\left(\delta y_{f}^{\prime}\right)^{2}$, which is the sum of following three terms.

First term:

$$
\begin{aligned}
& \left\langle\gamma_{f}\left(\delta y_{f, 1}\right)^{2}+2 \alpha_{f} \delta y_{f, 1} \delta y_{f, 1}^{\prime}+\beta_{f}\left(\delta y_{f, 1}^{\prime}\right)^{2}\right\rangle \\
& \quad=\frac{1}{4 E_{f}} \sum_{i} \frac{\delta E_{i}^{2}\left(e V_{c}\right)^{2} \beta_{i}\left\langle\theta_{i}^{2}\right\rangle}{E_{i}^{3}} .
\end{aligned}
$$

Second term:

$$
\begin{aligned}
& \left\langle\gamma_{f}\left(\delta y_{f, 2}\right)^{2}+2 \alpha_{f} \delta y_{f, 2} \delta y_{f}^{\prime}+\beta_{f}\left(\delta y_{f}^{\prime}\right)^{2}\right\rangle \\
& =\frac{1}{E_{f}} \sum_{q 1} \sum_{q 2} \sqrt{\beta_{q 1}} \sqrt{E_{q 1}} k_{q 1} \frac{-\delta E_{q 1}}{E_{q 1}} \\
& \quad \times \sqrt{\beta_{q 2}} \sqrt{E_{q 2}} k_{q 2} \frac{-\delta E_{q 2}}{E_{q 2}} \cos \left(\phi_{q 1}-\phi_{q 2}\right)\left\langle y_{q 1} y_{q 2}\right\rangle,
\end{aligned}
$$

where the indices $q 1$ and $q 2$ run through all quadrupole magnets, and from Eq. (22),

$$
\begin{aligned}
\left\langle y_{q 1} y_{q 2}\right\rangle= & \sqrt{\beta_{q 1} \beta_{q 2}} \frac{1}{\sqrt{E_{q 1} E_{q 2}}} \\
& \times \sum_{i<q 1, q 2} \beta_{i} \sin \phi_{i q 1} \sin \phi_{i q 2} E_{i}\left\langle\Theta_{i}^{2}\right\rangle \\
= & \sqrt{\beta_{q 1} \beta_{q 2}} \frac{1}{\sqrt{E_{q 1} E_{q 2}}} \\
& \times \sum_{i<q 1, q 2} \beta_{i} \sin \phi_{i q 1} \sin \phi_{i q 2} \frac{\left(e V_{c}\right)^{2}}{4 E_{i}}\left\langle\theta_{i}^{2}\right\rangle .
\end{aligned}
$$

Third term:

$$
\begin{aligned}
2\left\langle\gamma_{f} \delta y_{f, 1} \delta y_{f, 2}+\alpha_{f} \delta y_{f, 1} \delta y_{f, 2}^{\prime}+\alpha_{f} \delta y_{f, 1}^{\prime} \delta y_{f, 2}\right. \\
\left.+\beta_{f} \delta y_{f, 1}^{\prime} \delta y_{f, 2}^{\prime}\right\rangle \\
=2 \frac{1}{E_{f}} \sum_{q} \sqrt{\beta_{q}} \sqrt{E_{q}} k_{q} \frac{-\delta E_{q}}{E_{q}} \\
\quad \times \sum_{i} \sqrt{\beta_{i}} \sqrt{E_{i}} \frac{-\delta E_{i}}{E_{i}} \cos \phi_{i q}\left\langle\Theta_{i} y_{q}\right\rangle,
\end{aligned}
$$

where $y_{q}$ is given by Eq. (22) and 


$$
\left\langle\Theta_{i} y_{q}\right\rangle= \begin{cases}\sqrt{\beta_{q} \beta_{i}} \sqrt{E_{i} / E_{q}} \sin \phi_{i q}\left\langle\Theta_{i}^{2}\right\rangle & (i<q) \\ 0 & (i>q) .\end{cases}
$$

Here, let us assume all particles get the nominal energy gain along the linac, meaning $\delta E$ is constant for each particle. In other words, energy deviation of each particle in the beam is dominantly determined at injection. This approximation is good for the ILC main linac.

From Eq. (17), replacing $\left(\delta E_{i}\right)^{2}$ and $\left(\delta E_{q}\right)^{2}$ by energy spread $\sigma_{E}^{2}$,

$$
\begin{aligned}
\left\langle\left[(\gamma \epsilon)^{2}-\left(\gamma \epsilon_{0}\right)^{2}\right] /\left(\gamma \epsilon_{0}\right)\right\rangle & \\
\approx & \frac{\sigma_{E}^{2}}{4 m c^{2}} \sum_{i} \frac{\left(e V_{c}\right)^{2} \beta_{i}\left\langle\theta_{i}^{2}\right\rangle}{E_{i}^{3}}+\frac{\sigma_{E}^{2}}{4 m c^{2}} \\
& \times \sum_{q 1} \sum_{q 2} k_{q 1} k_{q 2} \beta_{q 1} \beta_{q 2} \cos \left(\phi_{q 1}-\phi_{q 2}\right) \frac{1}{E_{q 1} E_{q 2}} \\
& \times \sum_{i<q 1, q 2} \beta_{i} \sin \phi_{i q 1} \sin \phi_{i q 2} \frac{\left(e V_{c}\right)^{2}}{E_{i}}\left\langle\theta_{i}^{2}\right\rangle+\frac{2 \sigma_{E}^{2}(e V c)^{2}}{m c^{2}} \\
& \times \sum_{q} \beta_{q} k_{q} \frac{1}{E_{q}} \sum_{i<q} \beta_{i} \frac{1}{E_{i}} \cos \phi_{i q} \sin \phi_{i q}\left\langle\theta_{i}^{2}\right\rangle .
\end{aligned}
$$

This formula is suitable for numerical calculation to estimate expected emittance growth due to random cavity tilt angle, for a given design of a linac, using a computer.

Here, we derive a more simple expression with some approximations. We assume the linac lattice is uniform FODO (iteration of focusing quadrupole magnet-drift space-defocusing quadrupole magnet-drift space) along the linac, which means we can write

$$
k_{q}= \begin{cases}k_{F} & \text { (focusing quadrupole) } \\ k_{D} & \text { (defocusing quadrupole) }\end{cases}
$$

where $k_{F}$ and $k_{D}$ are constant. Then, averaging pairs of $(q 1, q 2)$,

$$
\begin{aligned}
k_{q 1} k_{q 2} \beta_{q 1} \beta_{q 2} & \rightarrow \frac{1}{4}\left(k_{F}^{2} \beta_{F}^{2}+2 k_{F} k_{D} \beta_{F} \beta_{d}+k_{D}^{2} \beta_{D}^{2}\right) \\
& =\frac{1}{4}\left(k_{F} \beta_{F}+k_{D} \beta_{D}\right)^{2}
\end{aligned}
$$

We make

$$
\begin{aligned}
\cos \left(\phi_{q 1}-\phi_{q 2}\right) \sin \phi_{i q 1} \sin \phi_{i q 2}= & \left(\cos \phi_{q 1} \cos \phi_{q 1}+\sin \phi_{q 1} \sin \phi_{q 1}\right)\left(\sin \phi_{i} \cos \phi_{q 1}-\cos \phi_{i} \sin \phi_{q 1}\right) \\
& \times\left(\sin \phi_{i} \cos \phi_{q 2}-\cos \phi_{i} \sin \phi_{q 2}\right) \rightarrow \cos ^{2} \phi_{q 1} \cos ^{2} \phi_{q 2} \sin ^{2} \phi_{i} \\
& +\sin ^{2} \phi_{q 1} \sin ^{2} \phi_{q 2} \cos ^{2} \phi_{i} \rightarrow 1 / 4
\end{aligned}
$$

where we took the average over many quadrupole magnet pairs and cavities, and replaced $\sin ^{2}$ or $\cos ^{2}$ by $1 / 2$ and $\sin \times \cos$ zero.

From similar consideration,

$$
\cos \phi_{i q} \sin \phi_{i q} \rightarrow 0
$$

averaging quadrupole magnets and cavities, and the third term of Eq. (29) vanishes.

For summation of the energy dependent factors, we approximate as follows:

$$
\sum_{i} \frac{1}{E_{i}^{3}} \rightarrow \frac{1}{e V_{c}} \int_{E_{0}}^{E_{f}} \frac{d E}{E^{3}}=\frac{1}{2 e V_{c}}\left(\frac{1}{E_{0}^{2}}-\frac{1}{E_{f}^{2}}\right)
$$

and

$$
\begin{aligned}
\sum_{q 1} \sum_{q 2} & \sum_{i<q 1, q 2} \frac{1}{E_{q 1} E_{q 2} E_{i}} \\
\approx & 2 \sum_{q 1} \sum_{q 2<q 1} \sum_{i<q 2} \frac{1}{E_{q 1} E_{q 2} E_{i}} \rightarrow 2 \frac{1}{\Delta_{E q}} \int_{E_{0}}^{E_{f}} \frac{d E_{q 1}}{E_{q 1}} \frac{1}{\Delta_{E q}} \\
& \times \int_{E_{0}}^{E_{q 1}} \frac{d E_{q 2}}{E_{q 2}} \frac{1}{e V_{c}} \int_{E_{0}}^{E_{q 2}} \frac{d E_{i}}{E_{i}}=\frac{1}{3 \Delta_{E q}^{2} e V_{c}}\left(\ln \frac{E_{f}}{E_{0}}\right)^{3},
\end{aligned}
$$

where $\Delta_{E q}$ is the energy gain between two quadrupole magnets, which is assumed to be constant along the linac.

Finally, collecting these approximations, we have a formula for expected emittance growth due to random cavity tilt:

$$
\begin{aligned}
\left\langle\left[(\gamma \epsilon)^{2}-\left(\gamma \epsilon_{0}\right)^{2}\right] /\left(\gamma \epsilon_{0}\right)\right\rangle & =\frac{e V_{c} \sigma_{E}^{2}}{8 m c^{2}} \bar{\beta}\left(\frac{1}{E_{0}^{2}}-\frac{1}{E_{f}^{2}}\right)\left\langle\theta^{2}\right\rangle+\frac{e V_{c} \sigma_{E}^{2}}{192 m c^{2} \Delta_{E q}^{2}} \\
& \times \bar{\beta}\left(k_{F} \beta_{F}+k_{D} \beta_{D}\right)^{2}\left(\ln \frac{E_{f}}{E_{0}}\right)^{3}\left\langle\theta^{2}\right\rangle .
\end{aligned}
$$

The first term can be usually ignored compared with the second term, since $\Delta_{E q}^{2} \ll E_{0}^{2}$. Note that emittance growth is half of this, for small emittance growth. Then we obtain the approximate formula, in the case $\Delta \epsilon=\epsilon-\epsilon_{0} \ll \epsilon_{0}$,

$$
\langle\Delta(\gamma \epsilon)\rangle \approx \frac{e V_{c}\left(\sigma_{E}\right)^{2}}{384 m c^{2} \Delta_{E q}^{2}} \bar{\beta}\left(k_{F} \beta_{F}+k_{D} \beta_{D}\right)^{2}\left(\ln \frac{E_{f}}{E_{0}}\right)^{3}\left\langle\theta^{2}\right\rangle
$$




\section{EFFECT OF MISALIGNMENT OF QUADRUPOLE MAGNET}

\section{A. Orbit due to random offset of many quadrupole magnets}

In this section, we estimate orbit change and emittance growth due to misalignment of quadrupole magnets, similarly to the estimation of cavity tilt effects in the previous section.

The transverse orbit angle change of a particle with nominal energy at one quadrupole magnet is

$$
\Theta_{j}=-a_{j} k_{j}
$$

where $a_{j}$ is misalignment (offset) of the $j$ th quadrupole magnet and $k_{j}$ is the integrated strength of the magnet. Then, at the end of linac,

$$
\begin{aligned}
& y_{f, q}=\sum_{j} \sqrt{\beta_{f} \beta_{j}} \sin \phi_{j} \sqrt{E_{j} / E_{f}} a_{j} k_{j}, \\
& y_{f, q}^{\prime}=\sum_{j} \sqrt{\beta_{j} / \beta_{f}}\left(\cos \phi_{j}-\alpha_{f} \sin \phi_{j}\right) \sqrt{E_{j} / E_{f}} a_{j} k_{j},
\end{aligned}
$$

where the summation is taken for all quadrupole magnets, $\beta_{j}$ the beta function at the $j$ th quadrupole magnet, $\sin \phi_{j}$ phase advance between the quadrupole magnet to the linac end, and $E_{j}$ the beam energy at the quadrupole magnet.

Assuming $a_{j}$ is random and independent for each magnet, the expected (average) action at the linac end is

$$
\left\langle J_{f, q}\right\rangle=\frac{1}{2 E_{f}} \sum_{j} \beta_{j} k_{j}^{2} E_{j}\left\langle a_{j}^{2}\right\rangle .
$$

This formula is suitable for numerical calculation to estimate expected orbit due to offset misalignment of quadrupole magnets, for a given design of a linac, using a computer.

For a long linac, from similar consideration as in the previous section,

$$
\begin{aligned}
& \beta_{j} k_{j}^{2} \rightarrow \frac{\beta_{F} k_{F}^{2}+\beta_{D} k_{D}^{2}}{2}, \quad\left\langle a_{j}^{2}\right\rangle \rightarrow\left\langle a^{2}\right\rangle, \\
& \sum_{j} E_{j} \rightarrow \frac{1}{\Delta_{E q}} \int_{E_{0}}^{E_{f}} d E E=\frac{E_{f}^{2}-E_{0}^{2}}{2 \Delta_{E q}},
\end{aligned}
$$

we used Eq. (30), and assumed all magnets have the same expected square of misalignment $\left\langle a^{2}\right\rangle$.

Then,

$$
\left\langle J_{f, q}\right\rangle \approx \frac{1}{8 E_{f} \Delta_{E q}}\left(\beta_{F} k_{F}^{2}+\beta_{D} k_{D}^{2}\right)\left(E_{f}^{2}-E_{0}^{2}\right)\left\langle a^{2}\right\rangle .
$$

For comparing with normalized emittance

$$
\gamma\left\langle J_{f, q}\right\rangle \approx \frac{1}{8 m c^{2} \Delta_{E q}}\left(\beta_{F} k_{F}^{2}+\beta_{D} k_{D}^{2}\right)\left(E_{f}^{2}-E_{0}^{2}\right)\left\langle a^{2}\right\rangle .
$$

Here, we have obtained a simple formula for expected orbit change due to random offset of quadrupole magnets.

\section{B. Emittance growth due to random offset of many quadrupole magnets}

Here, we will derive expressions for emittance growth from dispersive effect, due to random offset of many quadrupole magnets.

We can use Eqs. (24)-(28), replacing sums over index $i$, which run through cavities, by sums over index $j$, which run through quadrupole magnets, and replacing $e V_{c} \theta_{i} / 2 E_{i}$ by $-a_{j} k_{j}$.

First term:

$$
\begin{aligned}
& \left\langle\gamma_{f}\left(\delta y_{f, 1}\right)^{2}+2 \alpha_{f} \delta y_{f, 1} \delta y_{f, 1}^{\prime}+\beta_{f}\left(\delta y_{f, 1}^{\prime}\right)^{2}\right\rangle \\
& \quad=\frac{1}{E_{f}} \sum_{j} \frac{\delta E_{j}^{2} \beta_{j} k_{j}^{2}\left\langle a_{j}^{2}\right\rangle}{E_{j}},
\end{aligned}
$$

where the index $j$ runs through all quadrupole magnets.

Second term:

$$
\begin{aligned}
& \left\langle\gamma_{f}\left(\delta y_{f, 2}\right)^{2}+2 \alpha_{f} \delta y_{f, 2} \delta y_{f}^{\prime}+\beta_{f}\left(\delta y_{f}^{\prime}\right)^{2}\right\rangle \\
& =\frac{1}{E_{f}} \sum_{q 1} \sum_{q 2} \sqrt{\beta_{q 1}} \sqrt{E_{q 1}} k_{q 1} \frac{-\delta E_{q 1}}{E_{q 1}} \\
& \quad \times \sqrt{\beta_{q 2}} \sqrt{E_{q 2}} k_{q 2} \frac{-\delta E_{q 2}}{E_{q 2}} \cos \phi_{q 1}-\phi_{q 2}\left\langle y_{q 1} y_{q 2}\right\rangle,
\end{aligned}
$$

where the indices $q 1$ and $q 2$ run through all quadrupole magnets, and

$$
\begin{aligned}
\left\langle y_{q 1} y_{q 2}\right\rangle= & \sqrt{\beta_{q 1} \beta_{q 2}} \frac{1}{\sqrt{E_{q 1} E_{q 2}}} \\
& \times \sum_{i<q 1, q 2} \beta_{i} \sin \phi_{i q 1} \sin \phi_{i q 2} E_{i}\left\langle\Theta_{j}^{2}\right\rangle \\
= & \sqrt{\beta_{q 1} \beta_{q 2}} \frac{1}{\sqrt{E_{q 1} E_{q 2}}} \\
& \times \sum_{j<q 1, q 2} \beta_{j} k_{j}^{2} \sin \phi_{j q 1} \sin \phi_{j q 2} E_{j}\left\langle a_{j}^{2}\right\rangle .
\end{aligned}
$$

Third term:

$$
\begin{aligned}
2\left\langle\gamma_{f} \delta y_{f, 1} \delta y_{f, 2}+\alpha_{f} \delta y_{f, 1} \delta y_{f, 2}^{\prime}+\alpha_{f} \delta y_{f, 1}^{\prime} \delta y_{f, 2}\right. \\
\left.+\beta_{f} \delta y_{f, 1}^{\prime} \delta y_{f, 2}^{\prime}\right\rangle \\
=2 \frac{1}{E_{f}} \sum_{q} \sqrt{\beta_{q}} \sqrt{E_{q}} k_{q} \frac{-\delta E_{q}}{E_{q}} \\
\quad \times \sum_{j} \sqrt{\beta_{j}} \frac{\delta E_{j}}{\sqrt{E_{j}}} \cos \phi_{j q}\left\langle\Theta_{j} y_{q}\right\rangle,
\end{aligned}
$$

where $\phi_{j q}$ is the betatron phase advance between the quadrupole magnets $j$ and $q$, and 


$$
\left\langle\Theta_{j} y_{q}\right\rangle= \begin{cases}\sqrt{\beta_{q} \beta_{j}} \sqrt{E_{j} / E_{q}} \sin \phi_{j q} k_{j}^{2}\left\langle a_{j}^{2}\right\rangle & (j<q) \\ 0 & (j>q) .\end{cases}
$$

Then, following the similar considerations in the previous section,

$$
\begin{aligned}
\left\langle\left[(\gamma \epsilon)^{2}-\left(\gamma \epsilon_{0}\right)^{2}\right] /\left(\gamma \epsilon_{0}\right)\right\rangle \approx & \frac{\left(\sigma_{E}\right)^{2}}{m c^{2}} \sum_{j} \frac{\beta_{j} k_{j}^{2}\left\langle a_{j}^{2}\right\rangle}{E_{j}}+\frac{\left(\sigma_{E}\right)^{2}}{m c^{2}} \sum_{q 1} \sum_{q 2} k_{q 1} k_{q 2} \beta_{q 1} \beta_{q 2} \cos \left(\phi_{q 1}-\phi_{q 2}\right) \frac{1}{E_{q 1} E_{q 2}} \\
& \times \sum_{j<q 1, q 2} \beta_{j} k_{j}^{2} \sin \phi_{j q 1} \sin \phi_{j q 2} E_{j}\left\langle a_{j}^{2}\right\rangle+\frac{2 \sigma_{E}^{2}}{m c^{2}} \sum_{q} \beta_{q} k_{q} \frac{1}{E_{q}} \sum_{j<q} \beta_{j} k_{j}^{2} E_{j} \cos \phi_{j q} \sin \phi_{j q}\left\langle a_{j}^{2}\right\rangle .
\end{aligned}
$$

This formula is suitable for numerical calculation to estimate expected emittance growth due to random cavity tilt angle, for a given design of a linac, using a computer.

Here, we derive a more simple expression with the same approximations as in the previous section. We use Eqs. (30)-(32), replacing $i$ by $j$. The third term vanishes as in the previous section.

The sum of the energy dependent factors in the second term can be approximated as

$$
\begin{aligned}
\sum_{q 1} \sum_{q 2} & \sum_{j<q 1, q 2} \frac{E_{j}}{E_{q 1} E_{q 2}} \\
\approx & 2 \sum_{q 1} \sum_{q 2<q 1} \sum_{j<q 2} \frac{E_{j}}{E_{q 1} E_{q 2}} \rightarrow \frac{2}{\Delta_{E q}^{3}} \int_{E_{0}}^{E_{f}} \\
& \times \frac{d E_{q 1}}{E_{q 1}} \int_{E_{0}}^{E_{q 1}} \frac{d E_{q 2}}{E_{q 2}} \int_{E_{0}}^{E_{q 2}} d E_{j} E_{j} \\
= & \frac{E_{f}^{2}-E_{0}^{2}\left[2\left(\ln \frac{E_{f}}{E_{0}}\right)^{2}+2\left(\ln \frac{E_{f}}{E_{0}}\right)+1\right]}{4 \Delta_{E q}^{3}} .
\end{aligned}
$$

Finally,

$$
\begin{aligned}
\left\langle\left[(\gamma \epsilon)^{2}-\left(\gamma \epsilon_{0}\right)^{2}\right] /\left(\gamma \epsilon_{0}\right)\right\rangle & \\
\approx & \frac{\left(\sigma_{E}\right)^{2}\left(\beta_{f} k_{f}^{2}+\beta_{d} k_{d}^{2}\right)}{2 m c^{2} \Delta_{E q}}\left(\ln \frac{E_{f}}{E_{0}}\right)\left\langle a^{2}\right\rangle \\
& +\frac{\left(\sigma_{E}\right)^{2} \bar{\beta}\left(k_{F} \beta_{F}+k_{D} \beta_{D}\right)^{2}}{16 m c^{2} \Delta_{E q}^{3}} \\
& \times\left\{E_{f}^{2}-E_{0}^{2}\left[2\left(\ln \frac{E_{f}}{E_{0}}\right)^{2}+2\left(\ln \frac{E_{f}}{E_{0}}\right)+1\right]\right\}\left\langle a^{2}\right\rangle .
\end{aligned}
$$

The first term can be usually ignored compared with the second term, since $\Delta_{E q}^{2} \ll E_{f}^{2}$. Emittance growth is half of this, for small emittance growth. Then we obtain the approximate formula, in the case $\Delta \epsilon=\epsilon-\epsilon_{0} \ll \epsilon_{0}$,

$$
\begin{aligned}
\langle\Delta(\gamma \epsilon)\rangle \approx & \frac{\left(\sigma_{E}\right)^{2}}{8 m c^{2} \Delta_{E q}^{3}} \frac{\bar{\beta}\left(k_{F} \beta_{F}+k_{D} \beta_{D}\right)^{2}}{4}\left\{E_{f}^{2}\right. \\
& \left.-E_{0}^{2}\left[2\left(\ln \frac{E_{f}}{E_{0}}\right)^{2}+2\left(\ln \frac{E_{f}}{E_{0}}\right)+1\right]\right\}\left\langle a^{2}\right\rangle .
\end{aligned}
$$

Here, we have obtained a formula for expected emittance growth due to random offset of quadrupole magnets.

\section{COMPARISON WITH TRACKING SIMULATION}

For checking validity of the derived formulas, tracking simulations are performed for the ILC main linac. The simulation code SLEPT [8] was used. Relevant parameters of the ILC main linac are listed in Table II [9]. For simplicity, we make the linac to be straight, while the actual ILC main linac is designed to be curved following earth's curvature. For taking average, simulation for each condition had been performed with 500 different sets of random numbers (random seeds).

The following figures compare results of tracking simulations and the analytic formulas [Eqs. (15), (36), (43), and (51)], for orbit and emittance along the linac, not only at the end of the linac.

First, we set the random tilt angle of cavities with RMS (root mean square) $15 \mu \mathrm{rad}$. Figure 1 shows the average of action, $J_{y}$, as a function of beam energy along the linac, obtained from the formula Eq. (15) and from the tracking simulation. Figure 2 shows normalized emittance as a function of beam energy along the linac, obtained from the formula Eq. (36) (the first term is ignored) and from the tracking simulation.

Then, we set the random quadrupole magnet offset with RMS (root mean square) $1 \mu \mathrm{m}$. Figure 3 shows the

TABLE II. Relevant parameters of the ILC main linac.

\begin{tabular}{lc}
\hline \hline$E_{0}$ & $15 \mathrm{GeV}$ \\
$E_{f}$ & $250 \mathrm{GeV}$ \\
$\sigma_{E}$ & $0.16 \mathrm{GeV}$ \\
$\left(\gamma \epsilon_{y}\right)$ & $2 \times 10^{-8} \mathrm{~m}$ \\
$\beta_{F, y}$ & $\sim 140 \mathrm{~m}$ \\
$\beta_{D, y}$ & $\sim 40 \mathrm{~m}$ \\
$k_{F}$ & $-0.0286 \mathrm{~m}^{-1}$ \\
$k_{D}$ & $0.0320 \mathrm{~m}^{-1}$ \\
$V_{c}$ & $\sim 32 \mathrm{MV} / \mathrm{m}$ \\
$\Delta_{E} q$ & $e V_{c} \times 26$ \\
\hline \hline
\end{tabular}




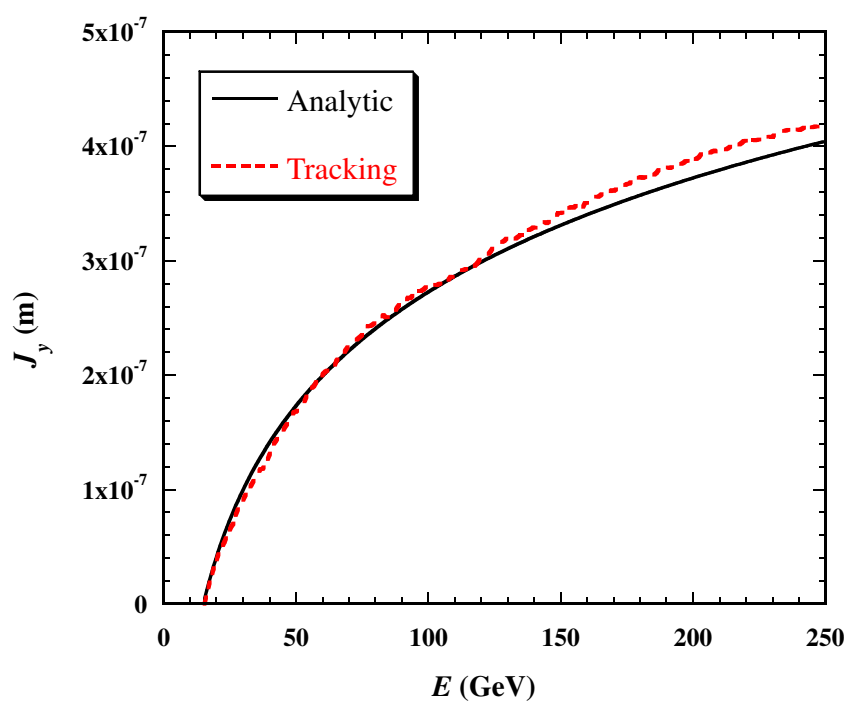

FIG. 1. Action induced by cavity tilt $15 \mu \mathrm{rad}$ (RMS), as a function of beam energy. Results of tracking simulation (solid line) and the analytic formula (dashed line) for the ILC main linac.

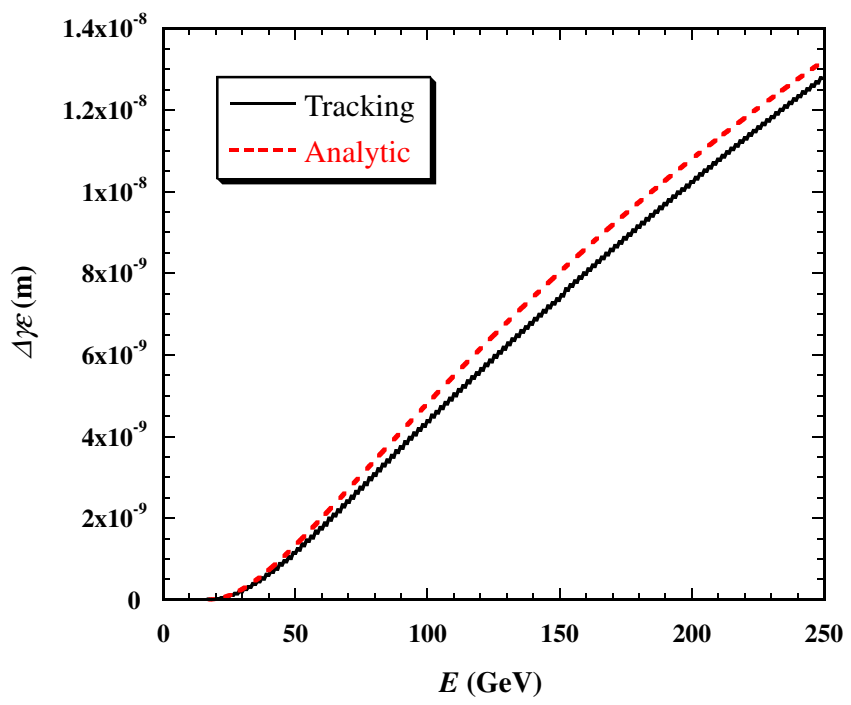

FIG. 2. Emittance growth induced by cavity tilt $15 \mu \mathrm{rad}$ (RMS), as a function of beam energy. Results of tracking simulation (solid line) and the analytic formula (dashed line) for the ILC main linac.

average of action, $J_{y}$, as a function of beam energy along the linac, obtained from the formula Eq. (43) and from tracking simulation. Figure 4 shows normalized emittance as a function of beam energy along the linac, obtained from the formula Eq. (51) (the first term is ignored) and from tracking simulation.

Since the final emittance growths in both cases are significant compared with the initial emittance, we did not use Eqs. (37) and (52).

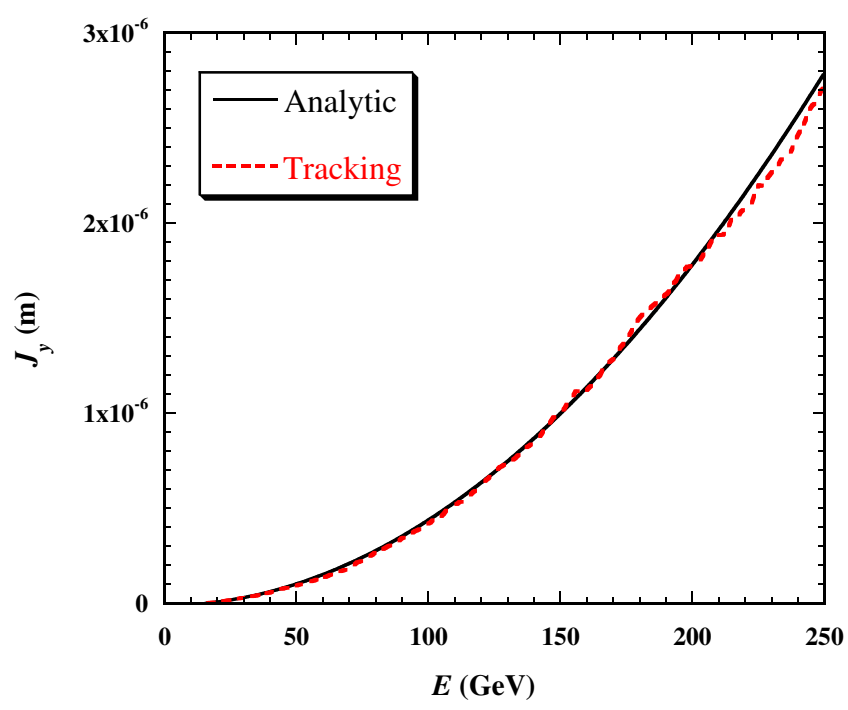

FIG. 3. Action induced by quadrupole magnet offset $1 \mu \mathrm{m}$ (RMS), as a function of beam energy. Results of tracking simulation (solid line) and the analytic formula (dashed line) for the ILC main linac.

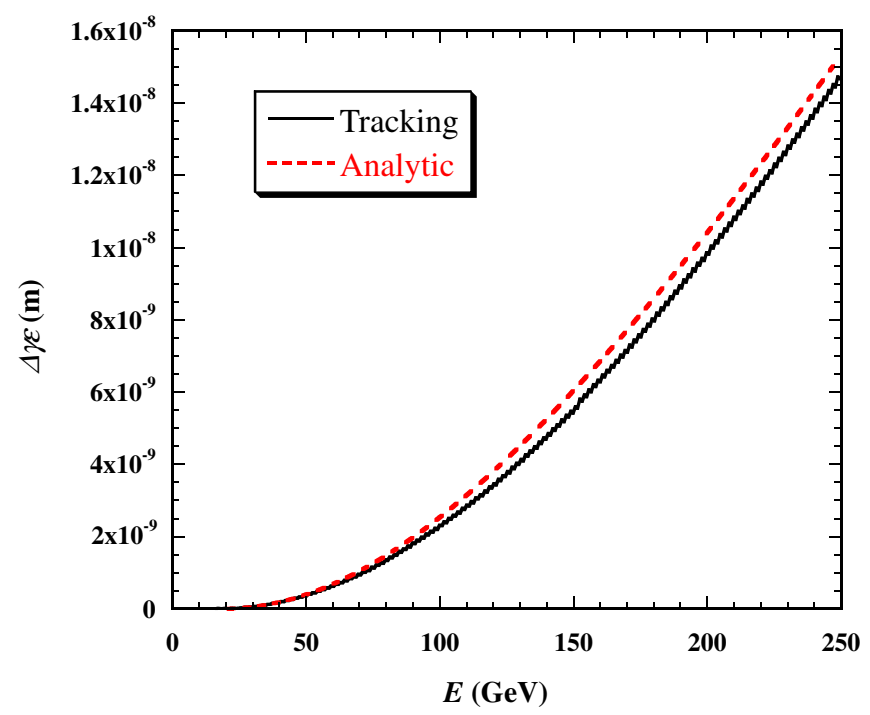

FIG. 4. Emittance growth induced by quadrupole magnet offset $1 \mu \mathrm{m}$ (RMS), as a function of beam energy. Results of tracking simulation (solid line) and the analytic formula (dashed line) for the ILC main linac.

Emittance growth dependence on the initial beam energy was also checked. Tracking simulations were performed for different initial energies $(15,30,50,100$, and $150 \mathrm{GeV}$ ) and the same final energy. (The linacs with higher initial energies are the same as the downstream part of the ILC main linac.) Figure 5 shows emittance growth as a function of initial beam energy with cavity tilt $15 \mu \mathrm{rad}$ (RMS). Figure 6 shows emittance growth as a function of initial beam energy with quadrupole magnet offset $1 \mu \mathrm{m}$ (RMS). 


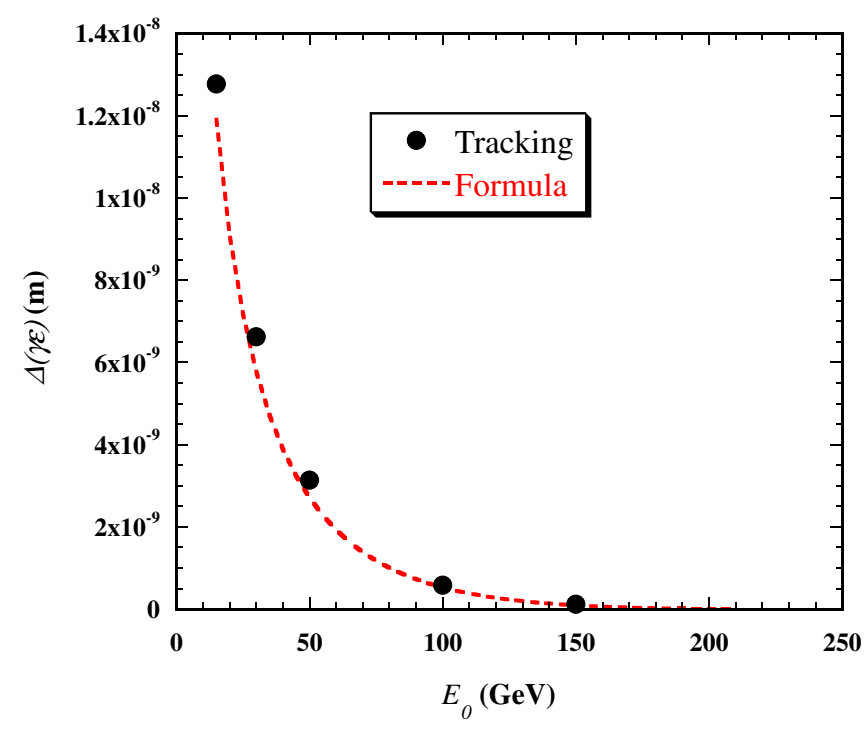

FIG. 5. Emittance growth induced by cavity tilt $15 \mu \mathrm{rad}$ (RMS) at the end of the linac (beam energy $250 \mathrm{GeV}$ ), as a function of initial beam energy. Results of tracking simulation (black circles) and the analytic formula (dashed line) for the ILC main linac.

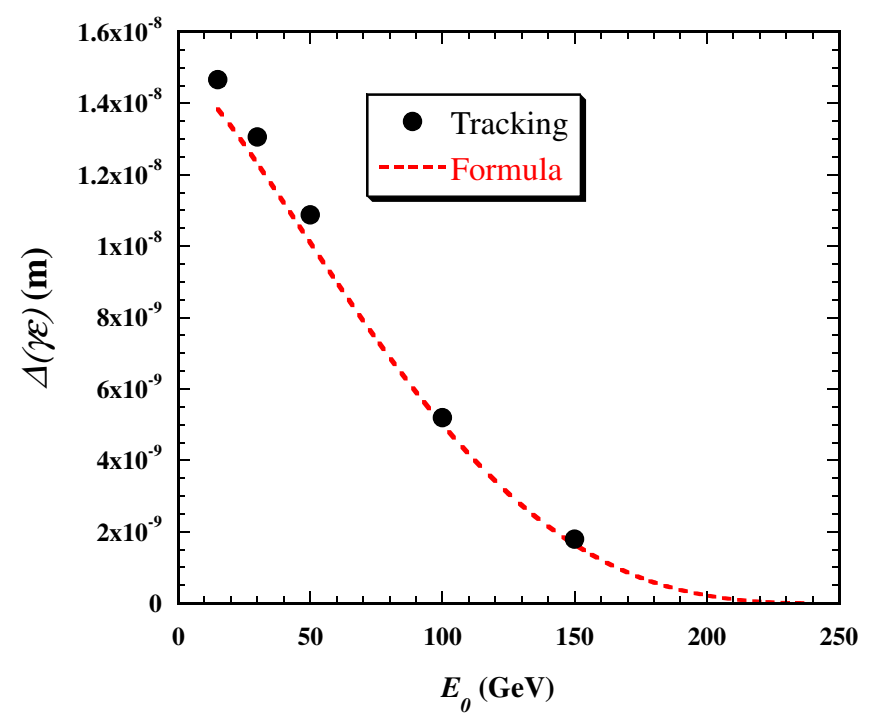

FIG. 6. Emittance growth induced by quadrupole magnet offset $1 \mu \mathrm{m}$ (RMS) at the end of the linac (beam energy $250 \mathrm{GeV}$ ), as a function of initial beam energy. Results of tracking simulation (black circles) and the analytic formula (dashed line) for the ILC main linac.

All of these results confirmed good agreement between the analytic formulas and the tracking simulations.

\section{SUMMARY AND DISCUSSIONS}

Formulas of orbit and emittance growth due to random tilting of accelerating cavities and random offset of quadrupole magnets are derived [Eqs. (15), (36), (37), (43), (51), and (52)]. The results are compared with tracking simulations, showing good agreement.

The formulas are valid for a linac with simple lattice design (see Table I). We also derived expressions for numerical calculations of orbit and emittance growth, which can be used for a linac with complicated lattice design [Eqs. (10), (29), (40), and (49)]. The calculations do not include the Monte Carlo method and will be much faster than tracking simulations.

Since orbit distortion and emittance growth due to static misalignment will be corrected using various techniques, the consideration of purely random errors here is more relevant for dynamic errors which are changing faster than any corrections. Effects of static errors can be assumed to be suppressed already, and effects of the errors considered here are regarded as a fast changing deviation from the static condition. Relevant errors will be mechanical vibrations of cavities and quadrupole magnets.

It should be noted that, if a cavity has a fixed tilting misalignment, change of accelerating voltage has an equivalent effect to mechanical change of the tilt angle [10]. For estimating the effects of such a change, we can make the replacement as

$$
\theta \rightarrow \theta_{\text {fix }} \Delta V / V,
$$

where $\Delta V / V$ is relative change of accelerating voltage and $\theta_{\text {fix }}$ is the fixed tilt angle.

[1] T. O. Raubenheimer, Phys. Rev. ST Accel. Beams 3, 121002 (2000).

[2] R. D. Ruth, in Proceedings of the U.S./CERN Joint Topical Course on "Frontiers of Particle Beams," South Padre Island, Texas, 1986 (Springer, New York, 1988).

[3] H. Henke, in Proceedings of the 1988 Linear Accelerator Conference, Continuous Electron Beam Accelerator Facility, Newport News, VA, 1988 (CERBAF-REPORT89-001;DE-AC05-84ER40150).

[4] G. Guignard and J. Hagel, Nucl. Instrum. Methods Phys. Res., Sect. A 434, 179 (1999).

[5] P. Tenenbaum, "Lecture in Second International Accelerator school for Linear Colliders," Ettore Majorana Center, Erice (Sicily), Italy, 2007.

[6] D. Schulte, "Lecture in Fifth International Accelerator School for Linear Colliders," Villars-Sur-Ollon, Switzerland, 2010.

[7] R. H. Helm, G. A. Loew, and W. K. H. Panofsky, in The SLAC Two-Mile Accelerator (W.A. Benjamin, Inc., New York, 1968), pp. 163-166.

[8] Simulation code SLEPT, http://lcdev.kek.jp/ kkubo/ reports/MainLinac-simulation/SLEPT/SLEPT-index.html.

[9] ILC Reference Design Report, http://www.linearcollider .org/about/publications/reference-design-report (2010).

[10] D. Schulte (private communication). 